[4] A. Ch. Chigogidze, Noncompact absolute extensors in dimension $n, n$-soft maps and their applications (in Russian), Izvestia AN SSSR, ser. matem. 50 (1986), 156-180.

[5] - Trivial bundles with fiber Tychonov cube (in Russian), Matem. Zametki 39 (1986), 747-756.

[6] - Uncountable powers of the line, the natural series and n-soft maps (in Russian), Dok1. AN SSSR 278 (1984), 50-53.

[7] - Characterization of dimension dim of $R$-compacta and universal spaces of given weight and given dimension (in Russian), Uspekhi Mat. Nauk 40: 5 (1985), 265-266.

[8] - Zero-dimensional open mappings which increase dimension, Comm. Math. Univ. Carolinae 24 (1983), 573-581.

[9] - On the dimension of increments of Tychonov spaces, Fund. Math. 111 (1981), 25-36.

[10] S. Ferry, The homeomorphism group of a compact Hilbert cube manifold is an ANR, Ann. of Math. 106 (1977), 101-119.

[11] W. E. Haver, Mappings between ANR's that are fine homotopy equivalences, Pacific J. Math. 58 (1975), 457-461.

[12] S. Sirota, On spectral representation of spaces of closed subsets of bicompacta (in Russian), Dokl. AN SSSR 181 (1968), 1069-1072.

[13] H. Torunczyy, On CE-images of the Hilbert cube and characterizing of $Q$-manifolds, Fund. Math. 106 (1980), 31-40.

[14] - Characterizing Hilbert space topology, Fund. Math. 111 (1981), 247-262.

[15] H. Toruńczyk and J. E. West, Fibrations and Bundles with Hilbert Cube Manifold Fibers, preprint, 1980.

[16] E. V. Shchepin, Topology of limit spaces of uncountable inverse spectrum (in Russian), Uspekhi Mat. Nauk 31: 5 (1976), 191-226.

[17] - On Tychonoff manifolds (in Russian), Dokl. AN SSSR 246 (1979), 551-554.

[18] - Functors and uncountable powers of compacta (in Russian), Uspekhi Mat. Nauk 36: 3 (1981), 3-62.

DEPARTMENT OF MECHANICS AND MATHEMATICS MOSCOW STATE UNIVERSITY

119899 Moscow

USSR

\section{The second Peano derivative as a composite derivative}

by

\author{
Richard J. O'Malley (Milwaukee, Wis.)
}

Abstract. Differentiable functions $f: R \rightarrow R$ which simultaneously have a second derivative in the Peano sense, $f_{2}$, and a second derivative in the composite sense, $\left(f^{\prime}\right)_{c}^{\prime}$, are investigated. It is shown that $\left\{x:\left(f^{\prime}\right)_{c}^{\prime}(x) \neq f_{2}(x)\right\}$ is a scattered set, i.e. a countable set not dense in any perfect set. As a corollary it follows that $f_{\mathbf{2}}$ is the derivative of $f^{\prime}$ in the composite sense.

1. One of the long outstanding problems concerning Peano derivatives is the lack of a precise description of in what sense an $(n+1)$ th Peano derivative can be considered as a derivative of the associated (n)th Peano derivative. In this paper we provide an answer to that problem in the case when $n=1$ and the derivative is taken in the composite sense. To make the presentation as readily intelligible as possible requires a little background information.

There is a wealth of information about certain aspects of the class of Peano derivatives. The interested reader should see for example the excellent survey [2]. It is also safe to say that all known properties of these functions are also properties of approximate derivatives, see [4], [7]. However, for approximately differentiable functions $f: R \rightarrow R$ and its approximate derivative, $g$, the following property is known to hold, [6],

For any fixed perfect set $P$, there is an open interval, $(a, b)$ having nonempty intersection with $P$, such that for any $x$ in $(a, b) \cap P$,

$$
\operatorname{limit}_{\substack{h \rightarrow 0 \\ x+h \in P}} \frac{f(x+h)-f(x)}{h}=g(x) .
$$

It is naturally reasonable to hope an analogous situation holds for the class of Peano derivatives. In [6], the above enclosed relationship for a pair of functions $f$ and $g$ was formalized by saying $f$ was compositely differentiable to $g$ and that $g$ was a composite derivative of $f$.

Using that terminology, we can rephrase the previously mentioned problem as: Does the $n$th Peano derivative compositely differentiate to the $(n+1)$ th Peano derivative?

Historically, Denjoy has provided partial answers to that problem, [1]. He established that if besides the $(n+1)$ th $P$-derivative, the $(n+2)$ th Peano is also assumed 
to exist, then the $n$th Peano derivative does indeed compositely differentiate to the $(n+1)$ th Peano derivative. If this condition on $n+2$ is removed, the enclosed relationship above can only be verified pointwise for a residual : ubset of $(a, b) \cap P$.

Here we take an alternate approach for the first and second Peano derivatives. Namely we assume that we have a function $f: R \rightarrow R$ which has a derivative, $f^{\prime}$, a second Peano derivative, $f_{2}$ and that in addition, $f^{\prime}$ has a composite derivative, $\left(f^{\prime}\right)_{c}^{\prime}$. We will also call this $\left(f^{\prime}\right)_{c}^{\prime}$ the second composite derivative of $f$. It would be natural to expect that $f_{2}=\left(f^{\prime}\right)_{c}^{\prime}$. Unfortunately, this is not the case in general. Indeed in [4] the authors have constructed examples where $f^{\prime}$ is even approximately differentiable and still the function $\left(f^{\prime}\right)_{a p}^{\prime} \neq f_{2}$. Based on the existence of such examples it is also natural to investigate whether the example of [4] can be modified to provide a counterexample to $f_{2}$ being a composite derivative of $f^{\prime}$. Clearly such an example requires the existence a perfect set $E$ such that $\left\{x:\left(f^{\prime}\right)_{c}^{\prime}(x) \neq f_{2}(x)\right\}$ is dense in $E$. We show this is an impossibility and obtain as a corollary that $f^{\prime}$ compositely differentiates to $f_{2}$ if $f^{\prime}$ has a composite derivative.

2. We will prove the following theorem:

THEOREM. Let $f: R \rightarrow R$ have a finite derivative $f^{\prime}$, a second Peano derivative $f_{2}$ and let $f^{\prime}$ have a composite derivative, $\left(f^{\prime}\right)_{c}^{\prime}$ for all $x$ in $R$. Then the set

$$
\left\{x: f_{2}(x) \neq\left(f^{\prime}\right)_{c}^{\prime}(x)\right\}
$$

is a scattered set, that is a countable $G_{\delta}$. (Equivalently, it is not dense in any perfect set.)

Proof. Assume the contrary. That is, assume that there is a perfect set $Q \neq \varnothing$ such that $W=\left\{x: f_{2}(x) \neq\left(f^{\prime}\right)_{c}^{\prime}(x)\right\}$ is dense in $Q$. Based on previously proven results in [3] and the definition of composite derivative we can make a number of preliminary statements and reductions.

(1) We may assume that $Q$ has been suitably chosen so. that for every $x \in Q$,

$$
\lim _{\substack{h \rightarrow 0 \\ x \rightarrow h \in Q}} \frac{f^{\prime}(x+h)-f^{\prime}(x)}{h}=\left(f^{\prime}\right)_{c}^{\prime}(x)
$$

That is, by reducing $Q$, if necessary, we may assume $f^{\prime}$ compositely differentiates to $\left(f^{\prime}\right)_{c}^{\prime}$ over $Q$.

(2) By results in [3], the function $f^{\prime}$ restricted to $Q$ is extendable to a differentiable function $g$.

(3) If we define $G_{(x)}=\int_{0}^{x} g(t) d t$, we obtain a twice differentiable function on $R$.

(4) Next we consider the new function $F(x)=f(x)-G(x)$.

(a) $F(x)$ has a derivative $F^{\prime}=f^{\prime}-g$, (b) $F(x)$ has a second derivative in the Peano sense

$$
F_{2}=f_{2}-g^{\prime},
$$

(c) $F^{\prime}(x)$ has a composite derivative $\left(F^{\prime}\right)_{c}^{\prime}=\left(f^{\prime}\right)_{c}^{\prime}-g^{\prime}$.

(5) For all $x$ in $Q, F^{\prime}(x)=f^{\prime}(x)-g(x)=0$ and $\left(F^{\prime}\right)_{c}^{\prime}(x)=0$ and in particular,

$$
\lim _{h \rightarrow 0} \frac{F(x+h)-F(x)}{h^{2} / 2}=F_{2}(x) \text {. }
$$

(6) Suppose $x_{0}$ is a proof of $Q$ at which $F_{2}\left(x_{0}\right) \neq 0$. Then, dependent on the sign of $F_{2}\left(x_{0}\right), F$ has a strict local maxima or minima at $x_{0}$. Since for any function the set of local maxima or minima points is countable we have that:

$$
\left\{x: F_{2}(x) \neq 0\right\} \cap Q \text { is countable. }
$$

However $\left\{x: F_{2}(x) \neq 0\right\} \cap Q=\left\{x: f_{2}(x) \neq g^{\prime}(x)\right\} \cap Q=\left\{x: f_{2}(x) \neq\left(f^{\prime}\right)_{2}^{\prime}(x)\right\} \cap Q$.

(7) By known properties of Peano derivatives we may assume $Q$ is nowhere dense; though our arguments following do not require that observation.

This ends the preliminary statements.

Now let $\varepsilon>0$ be given. At any point $x_{0}$ in $Q$ where $F_{2}\left(x_{0}\right)=0$ there is a $\delta\left(x_{0}\right)>0$ such that: $\left|F(x)-F\left(x_{0}\right)\right| \leqslant \varepsilon\left(x-x_{0}\right)^{2}$ whenever we have $0<\left|x-x_{0}\right|$ $<\delta\left(x_{0}\right)$. For each $n=1,2, \ldots$, we define

$$
A_{n}=\left\{x_{0}:\left|F(x)-F\left(x_{0}\right)\right| \leqslant \varepsilon\left(x-x_{0}\right)^{2} \quad \text { whenever } 0<\left|x-x_{0}\right|<x_{n}\right\} \cap Q .
$$

Since $F$ is continuous, $A_{n}$ is closed for each $n$. Further we have that $Q \backslash \bigcup_{n=1} A_{n}$ is a subset of $\left\{x: F_{2}(x) \neq 0\right\} \cap Q$, and is at most countable. Therefore by the Baire category theorem, there is an $N$ and $(a, b)$ such that $\varnothing \neq(a, b) \cap Q \subset A_{N}$. We may assume that $b-a<\frac{1}{n}$. Since we are assuming $\left\{x: F_{2}(x) \neq 0\right\}$ is dense in $Q$, we may select a point $x_{\infty}$ from $(a, b) \cap Q \subset A_{N}$ such that $F_{2}\left(x_{\infty}\right) \neq 0$ and without loss of generality also assume
(i) $x_{\infty}=0$,
(ii) $F\left(x_{\infty}\right)=0$
(iii) $F_{2}\left(x_{\infty}\right)>0$.

Further we will assume $x_{\infty}$ is a limit point of $Q$ from the right at least.

We have $\lim _{x \rightarrow 0}\left(\frac{F(x)}{x^{2}}\right)=\gamma>0,0 \in A_{N}$ which implies $\gamma \leqslant \varepsilon$. We claim we may assume that $\gamma<\varepsilon$. This follows from the argument that since $(a, b) \cap Q$ can be assumed to be perfect and $\left\{x: F_{2}(x)=0\right\}$ is residual in $(a, b) \cap Q$, the Baire class one function $F_{2}$ must have $F_{2}(x)=0$ at any point of relative continuity in $Q$. Hence, 
by making a further reduction, if necessary; we may assume that $F_{2}(x)<\varepsilon$ over $Q$. So $\gamma<\varepsilon$ can be assumed.

Hindsight into the upcoming arguments causes us to wish to choose three positive numbers $\alpha, \beta, \Gamma$ in such a way that the following hold simultaneously:

$$
\begin{array}{ll}
\beta=\gamma+\Gamma, & 0<\Gamma<\sqrt{\gamma^{2}+\varepsilon^{2}}-\varepsilon, \\
\alpha=\gamma-\Gamma, & \beta<\varepsilon .
\end{array}
$$

We next determine a $\delta>0$ such that if $0<x<\delta<b$ then

$$
\text { * } a x^{2}<F(x)<\beta x^{2} \text {. }
$$

Inside the open interval $(0, \delta)$ we have for any $x_{0} \in A_{N}$ that

$$
-\varepsilon\left(x-x_{0}\right)^{2} \leqslant F(x)-F\left(x_{0}\right) \leqslant \varepsilon\left(x-x_{0}\right)^{2} ;
$$

so that

$$
F(x) \leqslant F\left(x_{0}\right)+\varepsilon\left(x-x_{0}\right)^{2}<\beta x_{0}^{2}+\varepsilon\left(x-x_{0}\right)^{2} .
$$

Since 0 is a right limit point of $A_{N}$ we may select an $x_{0}$ in $A_{N} \cap(0, f)$ so that $0<x_{0}\left(\frac{\varepsilon}{\varepsilon-\alpha}\right)<\delta$. Then at $\left(\frac{\varepsilon}{\varepsilon-\alpha}\right) x_{0}, * *$ above gives the inequality

$$
\begin{aligned}
F\left(\frac{\varepsilon}{\varepsilon-\alpha} \cdot x_{0}\right) & <\beta x_{0}^{2}+\varepsilon\left(\frac{\varepsilon}{\varepsilon-\alpha} \cdot x_{0}-x_{0}\right)^{2} \\
& =\beta x_{0}^{2}+\varepsilon x_{0}^{2}\left[\frac{\alpha}{\varepsilon-\alpha}\right]^{2}
\end{aligned}
$$

If we now consider the point value $\beta x_{0}^{2}+\frac{\varepsilon \cdot x_{0}^{2} \cdot \alpha^{2}}{(\varepsilon-\alpha)^{2}}$, we claim this value is less than $\alpha\left(\frac{\varepsilon}{\varepsilon-\alpha} x_{0}\right)^{2}$

Proof of claim.

$$
\beta+\frac{\varepsilon \alpha^{2}}{(\varepsilon-\alpha)^{2}}<\frac{\alpha \varepsilon^{2}}{(\varepsilon-\alpha)^{2}}
$$

if and only if

$$
\beta<\frac{\alpha \varepsilon(\varepsilon-\alpha)}{(\varepsilon-\alpha)^{2}}=\frac{\alpha \varepsilon}{\varepsilon-\alpha} .
$$

By our choices of $\alpha, \beta, \Gamma$ above we have:

$$
(\gamma+\Gamma)[\varepsilon-(\gamma-\Gamma)]=\varepsilon \gamma+\varepsilon \Gamma-(\gamma+\Gamma)(\gamma-\Gamma)<\varepsilon(\gamma-\Gamma) ;
$$

so substituting in $\alpha$ and $\beta$ and simplifying we have

$$
\beta<\frac{\varepsilon(\alpha)}{\varepsilon-\alpha} \text {. }
$$

This implies that

$$
F\left(\frac{\varepsilon}{\varepsilon-\alpha} x_{0}\right)<\alpha\left(\frac{\varepsilon}{\varepsilon-\alpha} x_{0}\right)^{2}
$$

however we have this point in $(0, \delta)$ and by * above this implies in turn that

$$
F\left(\frac{\varepsilon}{\varepsilon-\alpha} x_{0}\right)>\alpha\left(\frac{\varepsilon}{\varepsilon-\alpha} x_{0}\right)^{2}
$$

which is the contradiction we have been seeking.

COROLLARY. Under the hypothesis of the theorem above, the function $f^{\prime}$ has the second Peano derivative of $f$ as a composite derivative.

\section{References}

[1] A. Denjoy, Sur l'integration des coefficients differentials d'ordre supérieur, Fund. Math. 25 (1935), 273-326.

[2] M. J. Evans and C. E. Weil, Peano derivatives: A survey, Real Anal. Exchange 7 (1) (1981-82), $5-24$.

[3] M. Laczkovish, G. Petruska, Baire class one functions, approximately continuous functions and derivatives, Acta Math. Acad. Sci. Hungar. 25 (1974), 189-192.

[4] C. M. Lee and R. J. O'Malley, The second approximate derivative and the second approximate Peano derivative, Bull. Inst. Math. Acad. Sinica, 3 (1975), 193-197.

[5] R. J. O'Malley, Baire *1, Darboux functions, Proc. Amer. Math. Soc., 60 (1976), 187-192.

[6] - Decomposition of approximate derivatives, Proc. Amer. Math. Soc., 69 (1978), 243-247.

[7] R. J. O'Malley and C.E. Weil, Selective, bi-selective, and cumposite differentiation, Acta Math. Acad. Sci. Hungar. 43 (1984), 31-36.

\section{DEPARTMENT OF MATHEMATICS}

UNIVERSITY OF WISCONSIN-MILWAUKFE

Milwaukee, Wis. 5320

Received 7 January 1987;

in revised form 26 October 1987 